Physics International 2 (2): 50-56, 2011

ISSN 1948-9803

(C) 2011 Science Publications

\title{
Identification of Defects in Composite Materials Using an Improved Wavelet Analysis Algorithm
}

\author{
Yahiaoui Aicha, Si-Chaib Med and O.A. Chellil \\ University of Boumerdes, \\ F.S.I. Laboratory of Dynamics Motors and Vibroacoustics, Algeria
}

\begin{abstract}
Problem statement: The present work carries on the use of a method based on the wavelet transform to detect internal flaws of composite materials. The objective of this work consists in working out a data processing sequence of an ultrasonic signal identifying nearly flaws in composite laminate materials and estimating their position. Approach: The use of a numerical signal processing technique, based on the Fast Wavelet Transforms was applied. Results: The method was implanted and optimized for detection and classification of delamination and porosity flaws in manufactured materials. Since the information about the signal requires a large amount of computation time and resources, a technique was used to reduce the dimensions of the sampling signals. In Non-destructive evaluation of stratified composite materials, the identification of some defect features requires more recent and advanced methods than classical techniques. Notably, in thin composite materials, the reflected NDE ultrasonic signals were overlapping. As a result, the flaws evaluation was becoming unfeasible. Many works dedicated to advanced signal processing based on time-frequency analysis had been widely used In NonDestructive Evaluation (NDE) applications. To evaluate the nearly flaw detection of delamination and porosity enclosed in composite multilayer plate, the wavelet analysis was applied to ultrasound waveforms acquired by immersion pulse-echo technique. Conclusion: The obtained results offer some defect features relating their nature and position. The applied wavelet analysis provided excellent results for the investigated materials containing artificial delamination and porosity flaws.
\end{abstract}

Key words: Ultrasounds, wavelet transforms, signals, composites, nearly flaws, evaluation

\section{INTRODUCTION}

The design and analysis of composite materials being more complex and more expensive than the conventional materials, such as the steel and the aluminum, the anisotropic behaviour of these materials makes their structural analysis difficult, so the use of more sophisticated methods for their analysis is required (Med et al., 2009; Yahiaoui et al., 2009).

Developing the applications of composites in the mechanical structures requires the use of the non destructive evaluation of the mechanical behaviour of these materials. The field of the multilayer materials evaluation aims to strengthen the diagnosis in matter of researching defects especially the fibres basis composites of carbon or glass and epoxy (delamination, porosity, overlapping, bridging and inclusions) in particular, the distinction between micro-cavity and enriched areas with resin as well as the position of the echo signal resulting from the cavity near the first reflected echo signal. Let's note that the existence of defects in a composite can lead to a significant alteration of its mechanical characteristics. For the evaluation of reinforced fibre composites, emerges the problem of a great acoustic attenuation of the measuring signals. This problem raises a notorious difficulty in the applications of non destructive ultrasonic evaluating methods. As a result, it needs the use of different approaches of signal processing to analyse the signal of the propagating wave in the probed material. Some explicit methods based on the Discrete Wavelet Transform (DWT) have been proposed to solve this kind of problems. The basic algorithm used is based on the DWT to analyse the measurement errors of the size and to locate the position of the artificial defects in the tested composite materials.

The principal advantage of those approaches is that they allow a fine description of the local regularity of signals. The Fourier analysis did not make possible the connection of the local regularity of a function with the behaviour of its coefficients. The wavelet analysis allows a local description of the signals and obtaining new characterizations of the regularity. The wavelet decomposition of the majority of the signals reveals significant coefficients with only one small number of

(C) 2011 Yahiaoui Aicha, Si-Chaib Med and O.A. Chellil. This open access article is distributed under a Creative Commons Attribution (CC-BY) 3.0 license which permits unrestricted use, distribution, and reproduction in any medium, provided the original work is properly credited.

DOI: $10.3844 /$ pisp.2011.50.56 
positions in the time-scale plan, i.e., that the energy is preferentially localised at some frequencies and positions. The received ultrasonic signals are composed of the signal components and an additive Gaussian noise. So, the wavelet coefficients represent both the signal and the noise. To eliminate the noise, the best tool to use is the wavelet processing technique (Bilgutay et al., 1990; Cardoso and Saniie, 2003).

Concerning the position of the problem, this study consists in working out a data processing sequence of an ultrasonic signal for which would consist in the following steps:

- Detection of a flaw in a material

- Estimation of the exact position of the flaw in order to operate at the good place

- Simple and general modelling of the signals allowing a better analysis

- Identification of a signal by a well-known software, such as MATLAB

- Application of the wavelet transform for the timefrequency analysis to measure the thickness of a flaw (Demirli and Saniie, 2001)

\section{MATERIALS AND METHODS}

Methods of applications of the wavelet transform: The methods of wavelets anticipate powerful tools to analyze, quantify, compress, rebuild and model signals and images. They are useful to locally capture, identify and analyze processes, with multi-scales, non-stationary signals, to make it possible to explore aspects of data that other analysis techniques omit. A family of elementary functions is constructed by translation and dilation (or contraction) starting from a basic function, the mother or analyzing wavelet. There exist an infinite number of wavelets. The analyzing wavelet most often used, called Morlet wavelet or Mexican hat Fig. 1, is due to Morlet (Soman et al., 1993; Daubechies, 1990; Li et al., 1992; Mallat, 1989) and is given by Eq. 1:

$$
\psi(\mathrm{t})=\exp (\mathrm{ict}) \bullet \exp \left(-\frac{\mathrm{t}^{2}}{2}\right)
$$

We use a family of wavelets of functions $\psi^{\mathrm{a}, \mathrm{b}}$ indexed by two labels ( $\mathrm{a}$ and $\mathrm{b}$ ):

$$
\psi_{\mathrm{a}, \mathrm{b}}(\mathrm{t})=\frac{1}{\sqrt{\mathrm{a}}} \cdot \psi\left(\frac{\mathrm{t}-\mathrm{b}}{\mathrm{a}}\right)
$$

To remind, $\mathrm{a}$ and $\mathrm{b}$ are the translation and dilation or contraction parameters. The functions $\psi^{\mathrm{a}, \mathrm{b}}$ are called "wavelets"; the function $\psi$ is called "mother wavelet".

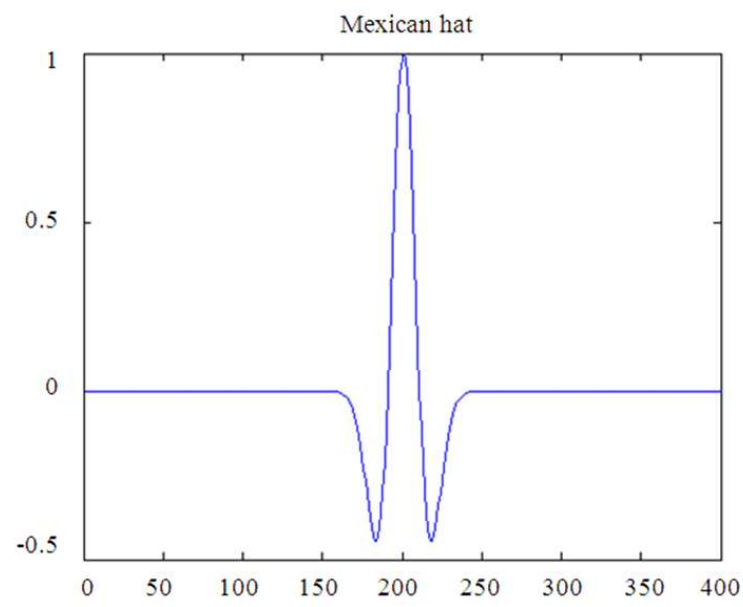

Fig. 1: Morlet wavelet

Note that $\psi$ is implicitly assumed to be real and if not, complex conjugate has to be introduced. As a changes, Eq. 2 with $b=0$ covers different frequency ranges (large values of a, correspond to small frequencies, while small values of a, correspond to high frequencies). This wavelet transform allows us to lead to a multi-resolution analysis. Changing the parameter $b$ as well allows moving the time localization centre: each $\mathrm{t}$ is localized around $\mathrm{t}=\mathrm{b}$. It follows that the wavelet transform provides a time-frequency description. The wavelet coefficients are then computed using the inner product of the analysed signal and the family of wavelets translated in time and dilated of the scale parameter a as follow. They are obtained by calculating the scalar product of the signal to be analyzed and the family of wavelets shifted in time and dilated or compressed by using the parameter a and b Eq. 3:

$\mathrm{S}(\mathrm{b}, \mathrm{a})=\frac{1}{\sqrt{\mathrm{a}}} \int_{0}^{\infty} \mathrm{s}(\mathrm{t}) \cdot \psi^{*}\left(\frac{\mathrm{t}-\mathrm{b}}{\mathrm{a}}\right) \mathrm{dt}$

where, $\mathrm{s}(\mathrm{t})$ is the signal to be analysed and $\psi^{*}$ the daughter wavelet.

\section{RESULTS}

Fast wavelet transform and applications:

Experimental Results: The detection of the flaws implies many factors, which influence the transmitted ultrasonic signal in the materials under investigation (Kazys et al., 2004; 2008; Bilgutay et al., 1979; Cavaccini et al., 2000). The acoustic theory of propagation in the materials shows that the parameters of the transmitting ultrasonic signal depend on many factors of which the most important are as follows: 
- Frequency and bandwidth of the ultrasonic signal

- Inspection of the trajectory and distance

- Position of flaws and their dimension

- Materials Properties

- The ultrasonic signals processing methods among which, the wavelet transform

The realization of the whole measurements is done by an ultrasonic bench including ultrasonic transducers with longitudinal waves, excited by a generator of ultrasounds transmitter-receiver. According to Fig. 2, control is done in a tank filled with water or any other medium ensuring a satisfactory coupling between the transducer and the part to be controlled and mobility with 3 directions of these transducers, so the possibility of examination of the complex parts by using a remotecontrolled manipulator arm. The signals are visualized on a numerical oscilloscope allowing their samplings. The unit is controlled by a micro-computer via a GPIB interface. Then the wavelet transform of the ultrasonic signal was analysed and the preliminary obtained results were presented. The training set was comprised of scanned signals from both damaged and undamaged samples using stratified composite materials. One dimensional wavelet transform is used to test our algorithm. This allowed us to study and identify the changes introduced in an ultrasound waveform by the presence of flaws. Scale-time plots were studied. Some flaws (delamination, porosity) in some composite materials were investigated for initial time-position of flaw peaks. Notice that when an ultrasound signal propagates through a material, the frequency components are modified. The composite materials themselves modify the frequencies: they degrade them like seen in the following Fig. 4 and 5. So the defects behave as frequency filters (Kazys et al., 2008; Cavaccini et al., 2000; Xin and Bilgutay, 1994; Saniie et al., 1990). The above problems confirm that testing composite materials require a fine frequency selection and a good signal interpretation.

The filtering action can be formulated as a change in transmission and reflection coefficients as follows (Murthy, 1992; Kaya, 1994):

- For low wavelength, transmission and reflection coefficients stay unchanged

- For high wavelength, the wave is completely transmitted, that is doesn't interact with the defect

- For intermediate wavelength, reflection /transmission coefficients decrease/increase linearly

Fast wavelet transform flowchart: The use of a straightforward Algorithm using a prohibitive number of points in a sample leads to a prohibitive computation time and a non-comprehensible decomposition.

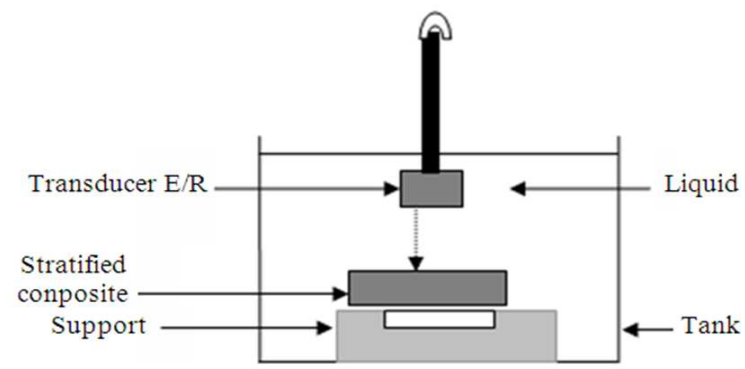

Fig. 2: Control by immersion in echo mode

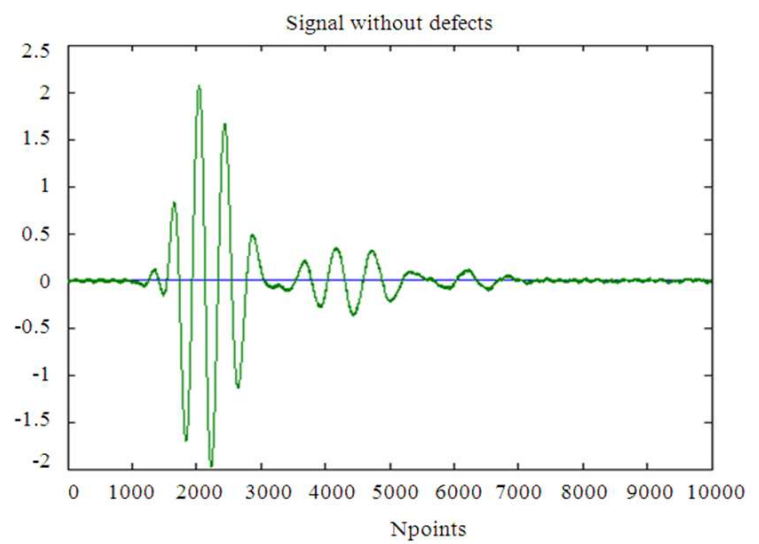

(a)

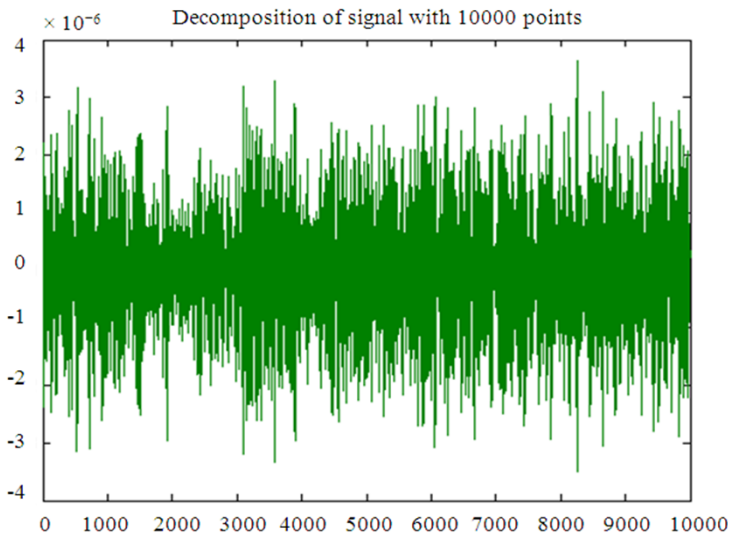

(b)

Fig. 3: (a) Measured signal without defects and (b) its decomposition

The first purpose of the proposed algorithm (Fig. 5) is a more effective computation procedure to compress the data sets needed for the calculation of the wavelet coefficients by simplifying the multidimensional (Kazys et al., 2008; Cavaccini et al., 2000) data sets to lower dimensions. This allowed us to achieve very high processing rate whose consequence is a better decomposition of a signal and a better interpretation of its graph. 


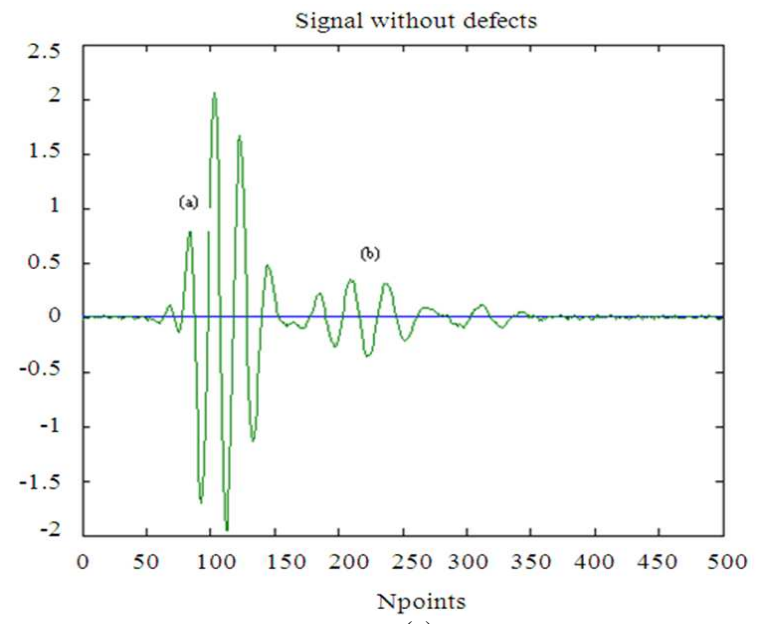

(a)

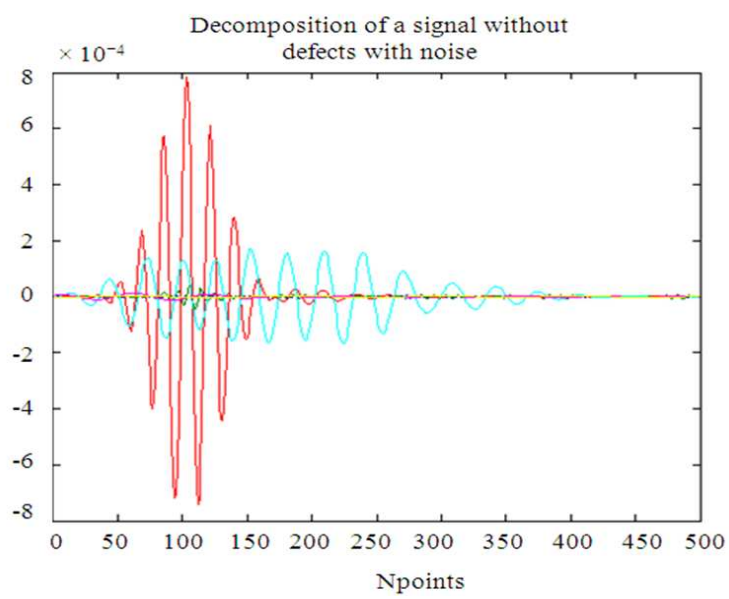

(b)

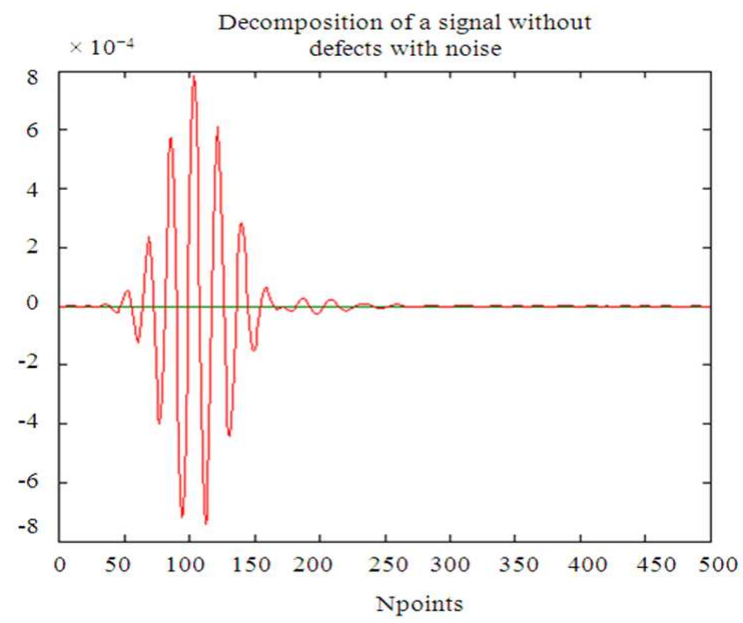

(c)

Fig. 4: (a) Measured Signal without Defects, (b) its decomposition with White Noise, (c) elimination of the white noise
The wavelet transform of $\mathrm{S}(\mathrm{t})$ is given by Eq. 3 where $\psi^{*}$ denotes the complex conjugate of $\psi$, a is tied to the frequency and b to the time (Fig. 4). To compute the coefficients, the real part of the Morlet wavelet is used (Soman et al., 1993; Daubechies, 1990).

\section{DISCUSSION}

Analysis and results discussion: The time-frequency analysis allows observing the nature of the signal whose parameters are unknown (rate of amplitude, frequency modulation). It also allows a global and a local analysis of the signal, i.e., a panoramic and a detailed analysis. The scalogram, by its positive character can assume the energy density function, which is not the case for a number of time-frequency representations (i.e. WignerVille representation which is a pseudo- representation of the energy since it can be negative).

A basic algorithm is used to compute the wavelet coefficients by means of numerical methods for obtaining results whose time and space complexities are more reduced. The results are exact and do not admit any approximations due to the truncation of the wavelet since the wavelet is taken on the same number of samplings.

The decomposed ultrasound signals for undamaged stratified composite material are shown in Fig. 3a, 3b and 3c. While in Fig. 6a and 6b, decompositions of damaged stratified composite materials are presented. For both materials, the front surface echo (a) and the front defect echo (b) can easily be detected. For the undamaged material, the noise could be eliminated by suppressing some wavelet coefficients not corresponding to any current frequency. So, the composite materials modify the frequency (they act like filters) and degrade the frequency.

When the material is undamaged, the oscillogram presents only oscillations due to the entering side (a) and those due to the bottom of the piece of material (b) (Fig. 3).

On the other hand, if there is a defect near the surface of the damaged material, the oscillogram presents two overlapped oscillations: echo of the surface of the investigated material and the one due to the defect (Fig. 7a and 7b).

Comparing with non-defect (Fig. 3) and defect signals (Fig. 7a and 7b) using the wavelet transform, the results of the decomposition show that specific signal characteristics appear only at defect regions. This provides that the amplitude of the waveform and the peak magnitude of frequency are parameters of defects (delamination, porosity) (Lee et al., 2007). 
Phy. Intl. 2 (2): 50-56, 2011

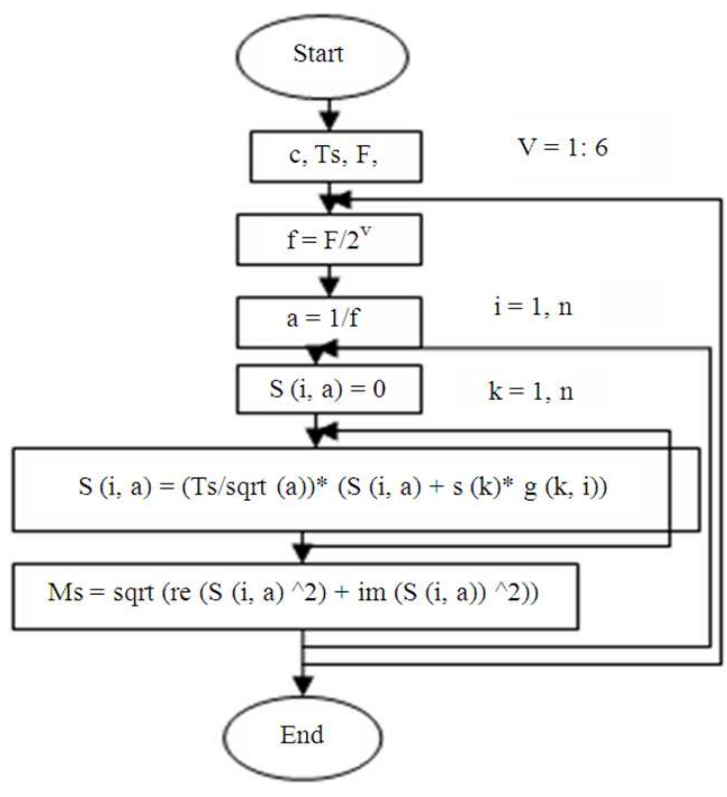

Fig. 5: Flowchart of the improved wavelet algorithm

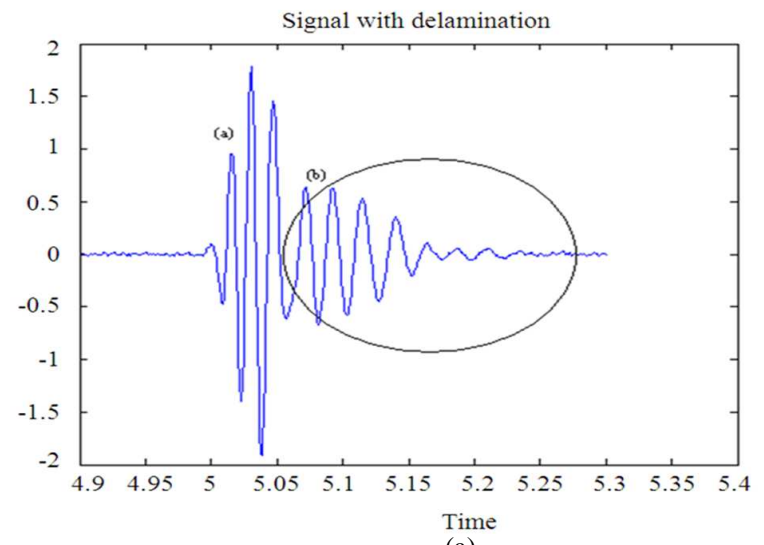

(a)

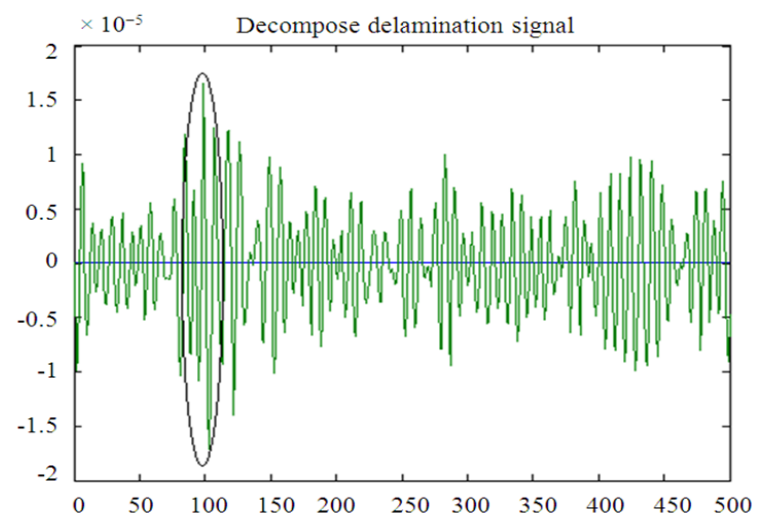

(b)

Fig. 6: (a) Ultrasound Measured Signal with Delamination Defects, (b) its Wavelet transform

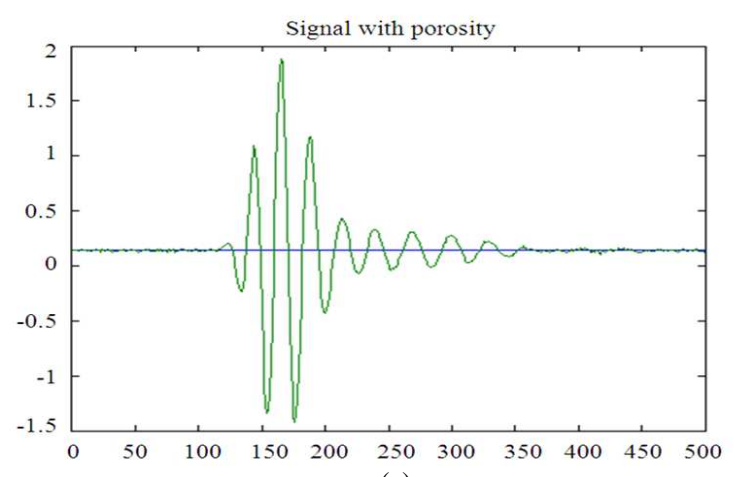

(a)

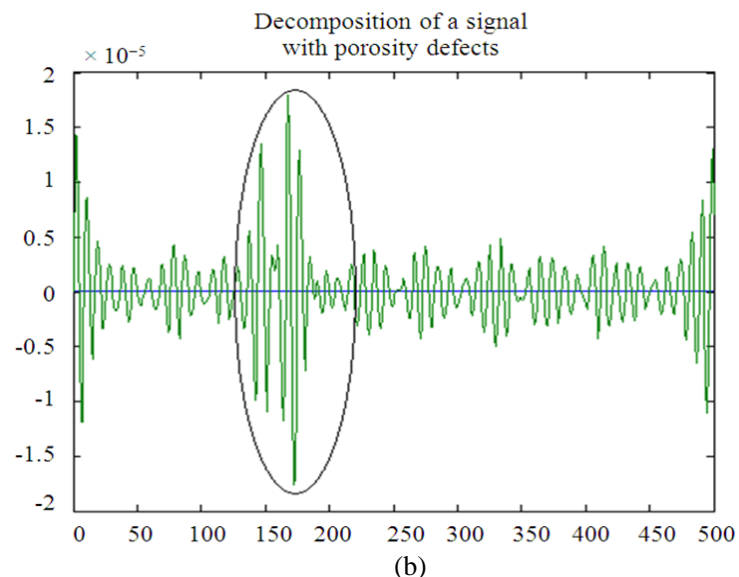

Fig. 7: (a) Ultrasound Measured Signal with Porosity Defect, (b) Its Wavelet transform

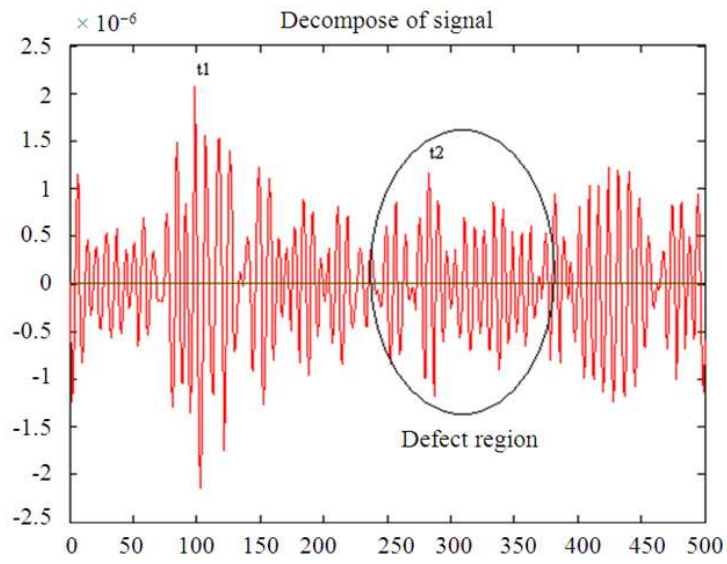

Fig. 8: Delamination defects

For the analysis of the artificial defect relating to the delamination, the default echo represented in the ultrasound signal, the experimental $\mathrm{s}_{1}(\mathrm{t})$ in Fig. 8 has been detected at $t_{2}$ while $t_{1}$ is the entering echo. 
Phy. Intl. 2 (2): 50-56, 2011

Table 1: Comparison of theoretical measures

\begin{tabular}{lccr}
\hline Measured defect & $\begin{array}{l}\text { Performed measurement } \\
\text { using the method }\end{array}$ & $\begin{array}{l}\text { Reference } \\
\text { measurement }\end{array}$ & \multicolumn{2}{c}{$\begin{array}{l}\text { Performed depth } \\
(\mathrm{mm})\end{array}$} \\
\hline Experimental $\mathrm{s}_{1}(\mathrm{t})$ & $\begin{array}{l}\tau=0.757 \mu \mathrm{sec} \\
\tau=0.4455 \mu \mathrm{sec}\end{array}$ & $\begin{array}{l}\tau=0.766 \mu \mathrm{sec} \\
\tau=0.475 \mu \mathrm{sec}\end{array}$ & $\mathrm{d}=1.482$ \\
Simulated $\mathrm{s}_{2}(\mathrm{t})$ & & $\mathrm{d}=0.872$ \\
\hline
\end{tabular}

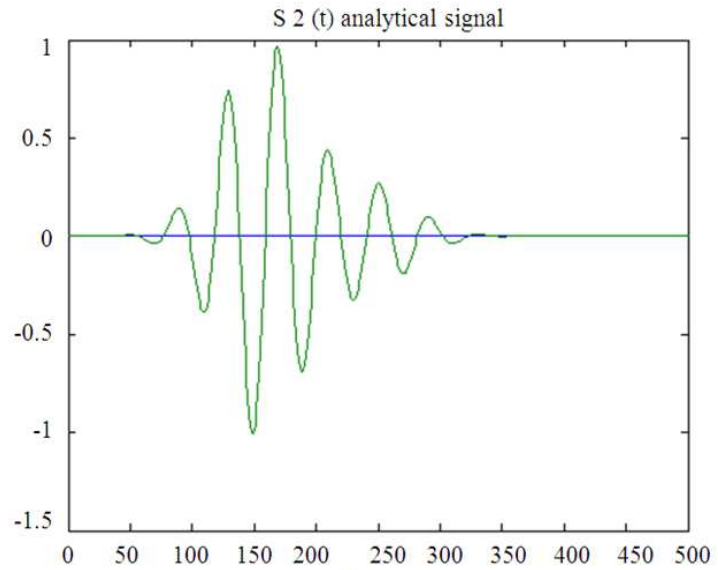

(a)

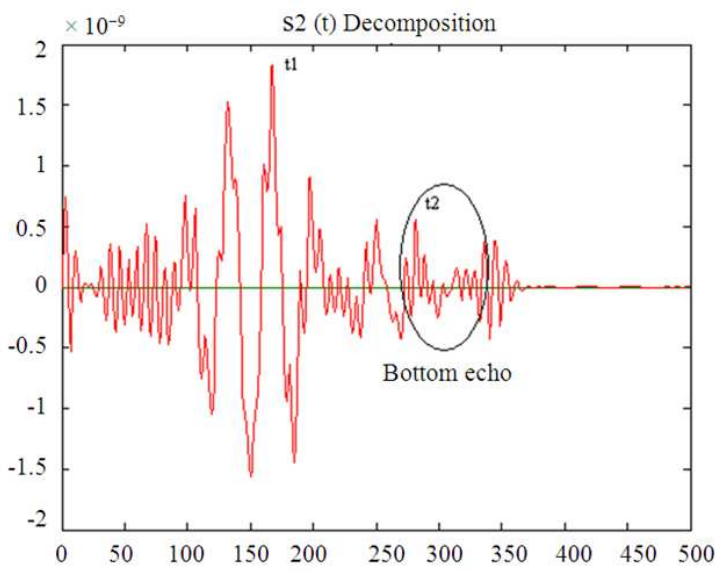

(b)

Fig. 9: An analytical signal without noise and its decomposition for the experimental signal

The time separating the entering echo and the defect echo denotes the time of flight. For $s_{2}(t)$, in Fig. 9, $\tau=$ $0.757 \mu \mathrm{s}$. The depth of the artificial defect is computed by Eq. 4:

$\mathrm{d}=\mathrm{V} \bullet \tau / 2$ And $\tau=\mathrm{t}_{2}-\mathrm{t}_{1}$

Where $\mathrm{V}$ is the velocity and $\tau$ represents the time of flight.

Taking $V=3.916^{\bullet} 10^{6} \mathrm{~mm} / \mathrm{s}, \mathrm{d}=1.482 \mathrm{~mm}$ for $\mathrm{s}_{1}$ (t). For theoretical validation, we presented an analytical signal $\left(\mathrm{s}_{2}(\mathrm{t})\right)$ that simulates an overlapping resulting from two signals: an interface signal and a reflected signal caused by an obstacle (bottom echo).
Performing the same calculations on $\mathrm{s}_{2}(\mathrm{t}), \tau=0.4455$ $\mu \mathrm{s}$ and $\mathrm{d}=0.872 \mathrm{~mm}$. The comparative results are shown in Table 1.

Compared to the referenced values, the error represents $4.2 \%$ in the first case and $6.21 \%$ in the second case. Those are quite good results.

\section{CONCLUSION}

The main objective of this work was to detect manufacturing defects and calculate their thicknesses using ultrasonic immersion technique. The use of a numerical signal processing technique, based on the FWT was applied. The method was optimized and implanted for detection and classification of delamination and porosity defects in composite materials. Since the information about the signal requires a large amount of computation time and resources, a technique to reduce the dimensions of the sampling signals was used. The implementation presented improves the time complexity of the basic algorithm by an exponential factor while compressing the data sample.

The scalogram characterizes the distribution of the signal energy in the time-frequency plane. In echograms, the wavelet analysis is useful for locating defects in some composite materials. A signal is emitted then received and the analysis of the perturbed area of the received signal allows to localize exactly the area of the defect, since having the velocity of the beam and the time the perturbation appears, the distance is just velocity times time. It would be interesting to establish in a practical way, in each domain a semantic better adapted to simplify interpretations of the results. The treatment of the signals by the wavelet transform makes it possible on one hand, to better understand the physical phenomena brought into action and on the other hand, to improve the ratio signal noise of the impact echo signals.

Starting from a visual inspection of ultrasonic echoes, delamination and porosity defectiveness can be illustrated. In case of delamination, the decomposed signal presents an initial peak initiated by the reflection of the ultrasonic signal on the medium surface and a second peak initiated by the reflection on the defect. While for porosity, the first peak is due to the reflection of the ultrasonic signal on the medium surface while the other peaks are generated by the reflection on the defect which, in this case is represented by multiple peaks. The peak amplitude in the case of porosity flaw is strongly lower than that of the delamination case. 


\section{REFERENCES}

Bilgutay, N.M., J. Saniie, E.S. Furgason and V.L. Newhouse, 1979. Flaw-Torain Echo Enhancement. Proc. Ultrasonics Int.

Bilgutay, N.M., R. Murthy, U. Bencharit and J. Saniie, 1990. Spatial processing for coherent noise reduction in ultrasonic imaging. J. Am. Stat. Assoc., 87: 728-736.

Cardoso, G. and J. Saniie, 2003. Data Compression and Noise Suppression of Ultrasonic NDE Signals Using Wavelets. Proceedings of the IEEE Symposium on Ultrasonics, 2003, Oct. 5-8, IEEE Xplore Press, pp: 250-253. DOI: 10.1109/ULTSYM.2003.1293400

Cavaccini, G., M. Agresti and G. Borzacchiello, 2000. An evaluation approach to NDT ultrasound processes by wavelet transform. Alenia Un'Azienda Finmeccanica-Pomigliano d'Arco Naples, Italy.

Daubechies, I., 1990. Ten Lectures on Wavelets. 1st Edn., Society for Industrial and Applied Mathematics, Philadelphia, ISBN-10: 0898712742, pp: 357 .

Demirli, R. and J. Saniie, 2001. Model-based estimation of ultrasonic echoes. Part II: Nondestructive evaluation applications. IEEE Transactions Ultrasonics, Ferroelectrics Frequency Control, 48: 803-811. DOI: 10.1109/58.920714

Kaya, O.K., 1994. Ultrasonic Target Detection using Wavelet Decomposition. 1st Edn., Drexel University, pp: 188 .

Kazys, R., D. Pagodinos and O. Tumsys, 2004. Application of the hilbert-huang signal processing to ultrasonic nondestructive testing of composite materials. ULTRAGARSAS, 50: 17-23.

Kazys, R., O. Tumsys and D. Pagodinos, 2008. A new ultrasonic technique for detection and location of defects in three-layer plastic pipes with a reinforced internal layer. ULTRAGARSAS (ULTRASOUND), 63: 19-27.
Lee, S.J., Y.J. Ha, J.H. Lee and J.H. Byun, 2007. Experimental Evaluation of Delamination in CFRP using laser-based Ultrasound, Prague, Czech Republic.

Li, X., N.M. Bilgutay and R. Murthy, 1992. Spectral histogram using the minimization algorithm-theory and applications to flaw detection. IEEE Trans. Ultrasonic Ferroelect. Frequency Control, 39: 279284. DOI: $10.1109 / 58.139126$

Mallat, S.G., 1989. A theory for multiresolution signal decomposition: The wavelet representation. IEEE Trans. Patt. Anal. Mach. Intell., 11: 674-693. DOI: 10.1109/34.192463

Med, O., N. Si-Chaib, A. Rezig, M.S. Yahiaoui and Y. Bouamrane et al., 2009. Nearly Detection and localisation by ultrasounds in thin composite plates by using the wavelet coefficients analysis. Congrés International, Conception et Modélisation des Systèmes Mécaniques, CMSM.

Murthy, R., 1992. Statistical characterization of Frequency Diverse Ultrasonic Signals. 1st Edn., Drexel University, pp: 246.

Saniie, J., K.D. Donohue and N.M. Bilgutay, 1990. Order statistic filters as postdetection processors. EEE Trans. Acoustics, Speech Signal Process., 38: 1722-1732. DOI: 10.1109/29.60103

Soman, A.K., P.P. Vaidyanathan and T.Q. Nguyen, 1993. Linear phase paraunitary filter banks: Theory, factorizations and designs. EEE Trans. Acoustics, Speech Signal Process., 41: 3480-3496. DOI: $10.1109 / 78.258087$

Xin, J. and N.M. Bilgutay, 1994. Detection and resolution of multiple targets using time-frequency techniques. Proceedings of the IEEE Ultrasonics Symposium, Oct. 31-Nov. 3, IEEE Xplore Press, Cannes, France, 2: 1133-1137. DOI: 10.1109/ULTSYM.1994.401788

Yahiaoui, A., M.O. Si-Chaib and M. Benantar, 2009. Contribution of the wavelet transforms to the analysis of the composites. Adv. Mater. Appli. Acoustics Vibration, Cairo, Egypt. 